\title{
El Juego Dramático. Una Estrategia para Mejorar La Expresión Oral
}

\section{The dramatic game. a strategy to improve oral expression}

Fecha de recepción: 23 de noviembre de 2017

Fecha de aprobación: 05 de febrero de 2018

Artículo de reflexión

\section{Resumen}

Este artículo reseña los resultados de una investigación desarrollada en la Institución Educativa Técnica Agropecuaria San Rafael del municipio de Rondón (Boyacá) en las sedes Granada Oriente y Páramo; mediante un trabajo reflexivo, a partir de un diagnóstico realizado mediante la aplicación de entrevistas y observación directa, evidenciando escaso dominio del lenguaje verbal y gestual utilizado en procesos comunicativos. Esta investigación es de tipo acción, con enfoque de corte cualitativo, teniendo como objetivo evaluar los cambios presentados en la expresión oral con la implementación del juego dramático comoestrategia didáctica en los estudiantes de grado segundo; estructuradas en tres fases: primera; identificar el problema y diseño de talleres; segunda; aplicación de talleres; tercera; análisis y evaluación de resultados. La metodología planteada; muestra resultados en cuanto al tono de vOz, incremento del vocabulario y expresiones corporales en situaciones comunicativas. Evidencia un progreso significativo en la expresión oral de la población objeto de estudio.

Palabras clave: Expresión, fluidez verbal, habilidad comunicativa, juego dramático, Oralidad.
*Institución Educativa Técnica Agropecuaria "San Rafael” - Boyacá,-Colombia lolysoler@gmail.com **Institución Educativa Técnica Agropecuaria "San Rafael" - Boyacá, Colombia leidylopezvargas@hotmail. com

***Universidad Pedagógica y Tecnológica de Colombia, Boyacá, - Colombia jorge.duarte@uotc.edu.co

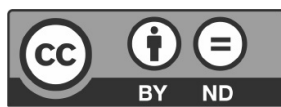




\section{Abstract}

This article reviews the results of a research carried out at "San Rafael" Educational Institution in the municipality of Rondón (Boyacá) in the Granada Oriente and Páramo branches; through reflective work, based on a diagnosis made through the application of interviews and direct observation, evidencing a lack of command of verbal and gestural language used in communicative processes. This action type research, with a qualitative approach, with the objective of evaluating the changes presented in the oral expression with the implementation of the dramatic game as a didactic strategy in the second-grade students; structured in three phases: first; identify the problem and design of workshops; second; application of workshops; third; Analysis and evaluation of results. The proposed methodology; shows results in terms of voice tone, vocabulary increase and body expressions in communicative situations. Evidence significant progress in the oral expression of the population under study.

Keywords: Expression, verbal fluency, communicative ability, dramatic play, Orality. 


\section{Introducción}

La investigación se realizó en dos escenarios de aprendizaje, uno ubicado en la zona rural, cuya sede se denomina Granada oriente; y otra en la zona urbana, denominada sede páramo, estas sedes pertenecen a la Institución Educativa Técnica Agropecuaria San Rafael del Municipio de Rondón. Teniendo en cuenta el diagnóstico realizado a los estudiantes, se planteó como objetivo de la investigación, evaluar los cambios en la expresión oral de los niños del grado segundo de las dos sedes en mención; con los estudiantes se aplicó el proceso metodológico, el cual se desarrolla en tres etapas: primera, identificación del problema, segunda; diseño de talleres y aplicación de las actividades, y la tercera que corresponde al análisis y sistematización de resultados.

En este sentido, el artículo aporta ideas de cómo hacer del aula de clase un entorno agradable, en donde a través de la expresión oral, el estudiante no sólo expresa su pensamiento y su sentir; sino que también reconstruya su herencia cultural y potencie su comunicación oral. Al explicitar la metodología y los resultados de la investigación, se aportan ideas que son útiles para algunos docentes, que desde su quehacer pedagógico buscan fortalecer en los alumnos una habilidad indispensable no sólo para el progreso académico del niño, sino también para su crecimiento como ser social, pues la buena expresión oral es un medio que determina el desenvolvimiento cotidiano de cada ser humano. También, se torna útil para el alumno quien a través de las actividades puede reconocer algunas formas de expresión que precisen en él una buena competencia en comunicación oral.

Se considera pertinente que las actividades encaminadas a estimular la destreza y afianzamiento de las habilidades comunicativas orales, estén orientadas de la práctica a la teoría, puesto que esta habilidad, como muchas otras, se desarrolla más fácil ejercitándolas; en otras palabras, a hablar, se aprende hablando. Es así que, a través del artículo se da cuenta de cómo - la puesta en práctica de una serie de actividades donde el niño tiene la posibilidad de expresar (práctica), se generan unas ideas que además de ser el resultado de una praxis, se tornan útiles para orientar ciertos procesos en el aula (teoría).

Por tal; razón, el artículo presenta una estructura donde se muestran unos postulados teóricos que evidencian, que la expresión oral es un proceso práctico e innato a través del cual se inicia a tejer el conocimiento y sigue estando presente en todos los procesos de aprendizaje y comunicación del ser humano; un estado del arte que muestra investigaciones previas que se han aplicado con el fin de motivar el desarrollo de la oralidad, pero que de ninguna manera tienen la misma ruta de aplicación de la presente investigación; una metodología que pone de manifiesto la ruta que se llevó a cabo para el desarrollo de la presente investigación; unos resultados que muestran lo que sucedió con las actividades aplicadas; y unas conclusiones que revelan las reflexiones y las ideas, luego del proceso y resultados que se obtuvieron.
Se considera pertinente que las actividades encaminadas a estimular la destreza y afianzamiento de las habilidades comunicativas orales, estén orientadas de la práctica a la teoría, puesto que esta habilidad, como muchas otras, se desarrolla más fácil ejercitándolas; en otras palabras, a hablar, se aprende hablando. 


\section{Marco histórico}

Para sustentar esta investigación; se hizo necesario indagar acerca de los trabajos que se han realizado en diferentes universidades del país sobre el tema en mención, entre las que se encuentran: la Universidad de la Amazonía; Universidad de Antioquia; Universidad Pedagógica Nacional y la Corporación Universitaria Minuto de Dios. De las diferentes consultas realizadas, se tuvo en cuenta las siguientes investigaciones.

Margot Cardozo Cuellar y Carlos Alberto Chicue Páez. Desarrollo de la expresión oral a través de los títeres, Universidad de la Amazonia. (2011). Esta investigación se llevó a cabo en el grado primero de la Institución Educativa Agroecológica Amazónico del municipio de Paujil. Cuyo propósito ha sido mejorar el proceso de enseñanza - aprendizaje de la expresión oral en los niños y niñas de la institución. Los principales resultados se reflejaron en la oralidad, con un buen tono de voz, el manejo de un buen vocabulario, fluidez verbal y entonación.

Otro aporte tomado del estudio realizado por María Emilia Núñez Rojas y Yenny Núñez Rojas titulado, "El teatrino como herramienta didáctica para el desarrollo de la expresión oral". Universidad de la Amazonia. (2012). Este proyecto se realizó en la Institución Educativa Juan Bautista Migani con estudiantes de grado transición. El objetivo es potenciar la expresión oral mediante el uso del teatrino como herramienta didáctica. Se destaca como resultado principal, el desarrollo de habilidades en los niños para hablar espontánea y creativamente a través de títeres.

Alina Navarro Gil. El teatro como estrategia para fortalecer la oralidad. Universidad de Antioquia. (2013). Desarrollado con estudiantes de grado quinto de la Institución Educativa Santo Cristo - sede Marco Fidel Suárez. Su objetivo, potenciar la oralidad, como elemento constitutivo de subjetividades, visión mítica del mundo y constituyente de identidad de los pueblos mediante el teatro. Como resultado, se puede decir que haber utilizado el teatro como herramienta y estrategia didáctica, contribuyó a mejorar la habilidad oral en aspectos de entonación, gestos y corporalidad, tal como se evidencia en los resultados obtenidos con los estudiantes del grado $5^{\circ}$ de la Institución Educativa Santo Cristo de Zaragoza.

Luis Jerónimo Muñoz y Luz Dary Parada. El teatro pedagógico como herramienta de desarrollo de las habilidades de expresión oral. Corporación Universitaria Minuto de Dios. (2015). Este proyecto de grado tuvo por objetivo indagar de qué manera el teatro pedagógico favorece las dinámicas de expresión en el aula. Los resultados obtenidos reflejan el aporte del teatro pedagógico al desarrollo de la expresión y comprensión, por ende, de las habilidades comunicativas en los estudiantes.

Leidy, J. Sánchez Bonilla. El teatro, actuación creativa, una estrategia para potenciar la competencia comunicativa. Universidad Pedagógica Nacional. (2013). En esta monografía se desarrolló en la Institución Tomás Carrasquilla con 
estudiantes de grado tercero, buscando potenciar la competencia comunicativa en los estudiantes a través de la actuación creativa. Como resultado, el teatro creativo permite al estudiante explorar sus habilidades comunicativas desde un contexto real, aporta a la creatividad y a la imaginación.

\section{Postulados teóricos}

Para significar, teorizar y dar sustento a la investigación, se tuvo en cuenta algunas teorías, que no solo orientaron la práctica investigativa y enriquecieron los saberes sobre el tema a trabajar, sino que permitieron argumentar adecuadamente los análisis y las conclusiones hasta el momento.

La expresión oral es una parte fundamental en el proceso de comunicación. Hablar es una condición imitativa social, por cuanto permite al ser humano darse a entender y llevar a cabo infinidad de procesos de socialización y de adquisición de conocimientos; dicha habilidad es inherente al hombre, no tiene en cuenta condición social, económica o académica, todos los seres humanos tienen la capacidad de relacionarse con sus semejantes a través de la expresión oral. Sin embargo; la formación académica, y la posición social y económica, sí influyen en el nivel y calidad del vocabulario que se emplea en las conversaciones.

Chomsky; (1997) citado por Barón, L \& Muller, O. (2014). En su investigación "La teoría lingüística de Noam Chomsky: del inicio a la actualidad; expone a través de su teoría gramática generativa, el estudio y desarrollo del carácter innato del lenguaje, afirmando que se nace con el lenguaje y con la capacidad de construir y comprender mensajes, lo cual permite pensar que el lenguaje necesita desarrollarse para llegar a su máxima expresión, que es la comunicación.

Gastón en su libro ¿Cómo hablar correctamente en público?, afirma que:

Es importante tener presente que quien tiene un buen repertorio oral, tiene más facilidades para plasmar sus pensamientos de forma escrita, por esta razón, la comunicación verbal presenta muchos problemas comunes con la expresión escrita, tales como la claridad, la precisión, el correcto uso del lenguaje, el orden, etc, que son cuestiones que afectan por igual uno a otro sistema de comunicación. (Gastón, 1997:15)

La expresión oral es la primera forma de apropiación del conocimiento, cuando el ser humano inicia su crecimiento físico e intelectual, el primer paso en el proceso de comunicación es a través del lenguaje, a partir de ese momento se empieza a adquirir saberes con la ayuda de la expresión oral, por medio de ésta: se comunica, se interroga, se expresan opiniones, se aclaran dudas, se dan y escuchan sugerencias, en fin, a partir de la expresión oral se inicia a tejer el conocimiento y sigue estando presente en todo el proceso de aprendizaje, no solo en los relacionados con la academia, sino en todas las actividades cotidianas.

El ser humano es un "ser sociable", que aprende en forma natural el proceso de la comunicación oral en el seno familiar, y como ser sociable tiene la
Es importante tener presente que quien tiene un buen repertorio oral, tiene más facilidades para plasmar sus pensamientos de forma escrita, por esta razón, la comunicación verbal presenta muchos problemas comunes con la expresión escrita, tales como la claridad, la precisión, el correcto uso del lenguaje, el orden, etc, que son cuestiones que afectan por igual uno a otro sistema de comunicación. 


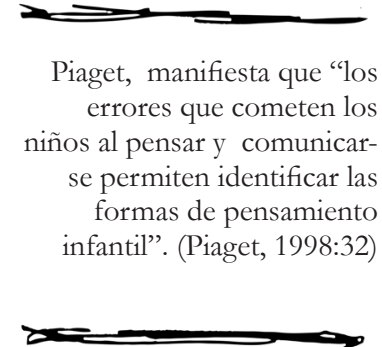

necesidad básica de estar en continuo intercambio de saberes, inquietudes, pensamientos, etc. Todos estos procesos se dan gracias a la utilización del diálogo de forma permanente; así como de un adecuado vocabulario que permita entender de forma clara, la intención comunicativa del interlocutor, proporcionando alcanzar los objetivos de la comunicación; ya sea mediante charlas, debates, conversatorios, foros, conferencias, entrevistas, diálogos, mesas redondas y otras tantas estrategias que permiten exteriorizar lo que se siente, piensa y desea mediante la utilización del habla.

Sandoval. (2007), afirma que:

Si bien; desde que nacemos socializamos los primeros años en nuestras familias, es en la escuela donde podemos habilitar nuestra calidad, ya que es de ahí donde aprendemos que existen reglas que nos permitirán utilizar nuestro lenguaje de forma adecuada y darle significado a lo que decimos. (Sandoval, 2007:13)

Para MiguelDe Zubiría, "la comunicación y expresión oral ocupan un lugar muy privilegiado en la transmisión de conocimientos y de cultura de una generación a otra". (Zubiría, 2007:11); esta es una de las razones por las cuales se ha decidido tener en cuenta dentro de los talleres a trabajar, las tradiciones culturales de la región, cumpliendo un doble propósito, el primero mejorar la expresión oral de los estudiantes mencionados; y el segundo, realizar un rescate cultural de coplas, poemas y canciones tradicionales.
Los procesos de comunicación han logrado grandes avances en todos los campos del conocimiento, quien se sabe comunicar es capaz de hacerse entender y tiene más posibilidades de alcanzar los objetivos o metas que se proponga; en cambio aunque tengan grandes metas, si no se tiene la capacidad de darlas a conocer de forma clara, es muy probable que no se alcancen, esto ocurre con los estudiantes; algunas veces tienen "la idea" o la respuesta a un interrogante, o desea expresar su opinión en diversos temas que lo afectan de forma positiva o negativa, sin embargo en ocasiones su escaso vocabulario o su timidez impiden logren transmitir lo que piensan, sienten o desean; interrumpiendo la adquisición de nuevos saberes.

Por las razones anteriormente expuestas, se desarrolló ésta investigación buscando mitigar los efectos negativos en cuanto a expresión y oralidad, del individuo en el campo de la educación infantil, para ser, implementadas como competencia y parte de la formación integral de manera libre y creativa.

Piaget, manifiesta que "los errores que cometen los niños al pensar y comunicarse permiten identificar las formas de pensamiento infantil". (Piaget, 1998:32)

Para desarrollar un buen proceso comunicativo, se debe tener en cuenta ciertos aspectos, algunos de estos son: expresar de forma clara lo que se desea comunicar, utilizando un tono de voz adecuado, haciendo uso de gestos que permitan dar a conocer con mayor claridad el mensaje, manejar un vocabulario variado, evitar las muletillas, 
jergas y palabras ambiguas. Para lograr los fines de la comunicación oral, se requiere conocer la población y determinar en qué momento del proceso de desarrollo del lenguaje y el pensamiento se encuentra para asegurar el asertividad del ejercicio investigativo.

Los estudiantes objeto de investigación se encuentran en el estadio preoperatorio o pre operacional; en esta etapa, los niños tienden a ser egocéntricos, sus conversaciones se conocen como monólogos colectivos, representa conceptos desarrollando y utilizando símbolos usualmente en forma de palabras. (Piaget, J, 1998)

En el campo de la educación, una de las características más importantes para el estudiante es el momento de expresar y de reflejar su alegría por medio de las diferentes actividades que desarrolla en el aula con sus compañeros.

Konstantin Stanislavsky. (1957); revela que el teatro se debe manejar con los niños de manera práctica y natural, con estilo esencial como si se estuviera actuando con adultos. De esta forma, el niño cuando está actuando va a sentir que esta representación es algo divertido, que está aprendiendo algo nuevo y que puede crear nuevas experiencias a manera de juego, a partir de la imaginación la cual implican saberes que maneja en su contexto escolar.

Motos y Tejado. (1998). Afirman que, con el teatro el estudiante desarrolla una competencia lingüística, corporal, plástica y musical, las cuales dan un enfoque integral en su formación del proceso de enseñanza- aprendizaje. En esta investigación, se utiliza el juego dramático, como estrategia didáctica; ya que con los estudiantes de primaria se puede trabajar dicha estrategia que permite la improvisación teniendo en cuenta un tema determinado, y no depende necesariamente de la memorización y la repetición de guion. Siguiendo este mismo ideal, analizamos a Magariño. (1996); quien afirma que el teatro se encuentra estrechamente vinculado con la representación y puesta en escena ante un público, mientras que el drama, tiene más que ver con "hacer y realizar" teniendo relación directa con el mundo de la enseñanza.

De igual manera, según Robles. (2009). El teatro mejora la entonación, el acento y el ritmo, además, de favorecer la inclusión de los estudiantes en las actividades de aula y su participación en las representaciones teatrales. Siendo esta una actividad que se pretende desarrollar en esta investigación. Así mismo, según Heladio Moreno Moreno. (1994), través del teatro el niño se introduce en un mundo fabuloso, con decoración y colorido. La participación de los sentidos en múltiples actividades creativas, da forma y estructura a la imaginación del infante, estimulando así su capacidad para crear, improvisar y cultivar el arte en toda su extensión. (Libro: "Teatro Infantil)

Del mismo modo, según Santamaría, A; Gómez, F; Ruiz, F; Boquete M; Vaqueiro, M; Peñasco, M; Moreda L; Comba O; Fernández, M \& Martín, H. (2014). En su artículo titulado "Arriba e 1 Telón"; enseñar teatro o enseñar desde el teatro y en si el uso del juego dramático; forma a los estudiantes de la lengua española en la práctica de las destrezas orales, mediante
Konstantin Stanislavsky. (1957); revela que el teatro se debe manejar con los niños de manera práctica y natural, con estilo esencial como si se estuviera actuando con adultos. 
la incorporación de estrategias inspiradas en el juego dramático. El juego dramático en el aula permite a los estudiantes aumentar el tiempo de práctica de juego para hacer las clases más dinámicas, activas y productivas; y así mejorar, de manera sensible, su pronunciación en español, sus habilidades comunicativas y su conocimiento de la realidad social y cultural.

\section{Metodología}

Basado en el trabajo Investigación Acción de Kunrt Lewis, en donde hace referencia a una amplia gama de estrategias para mejorar el sistema educativo y social y cuyo término fue utilizado por primera vez en 1994. Describe una forma de investigación que puede ligar el enfoque experimental de la ciencia social como programas de acción social, el cual respondiera a los problemas sociales de entonces. Mediante la investigación - acción, Lewis argumenta que se podía lograr en forma simultánea avances teóricos y cambios sociales (Kunrt L, 1994:3)

Esta investigación acción; se desarrolló desde un enfoque cualitativo. Utiliza preferentemente información interpretativa, descriptiva y no cuantificada. Tuvo en cuenta los procesos de conocimiento: se dio a conocer las causas que motivaron a los estudiantes a tomar ciertas actitudes y la influencia de estas en la convivencia escolar, el rendimiento académico; presentando así mismo los procesos de acompañamiento, que son las actividades que se realizaron para ayudar a solucionar el problema de la comunidad educativa. Se recopiló información a través de la aplicación de actividades lúdicas que requerían de hablar en público, recitar coplas, declamar poemas, interpretar canciones, dramatizar obras teatrales sencillas.

Finalmente, se realizó el análisis de los resultados obtenidos, estos se expresaron a través de un informe descriptivo del impacto de la propuesta, que encara el desafío de generar conocimiento con los actores sociales de una realidad, para que ellos asuman el poder de transformarla creativamente; ya que esta es una opción metodológica que permite que los investigadores reflexionen y centren la atención en la práctica participativa de la población. Para este caso, en la Institución Educativa Técnica Agropecuario San Rafael del municipio de Rondón en las sedes: rural Granada Oriente y urbana Páramo, la investigación cualitativa intenta hacer una aproximación global de las situaciones sociales para explorarlas, describirlas y comprenderlas de manera individual, a partir de los conocimientos que tienen las diferentes personas involucradas en ella.

La finalidad de esta investigación fue mejorar la expresión oral de los estudiantes participantes de este proyecto, mediante la aplicación de la estrategia de juego dramático. Para llevarla a cabo, se estructuraron las siguientes fases:

Fase uno (1): Identificar el problema y dimensionar el impacto que este causa en la comunicación asertiva de los estudiantes; elaboración de diagnóstico para identificar conocimientos previos acerca de la expresión oral por medio del juego dramático; a partir de los conocimientos previos y también de 
las dificultades encontradas, se diseñan talleres, haciendo uso de coplas, poesía, canciones, trabalenguas, retahílas, diálogo, mímicas, juego de roles, imitaciones, representaciones de actividades culturales y sociales propias de su entorno con el diseño del plan operativo a seguir en el proceso de investigación.

Fase dos (2): Ejecución de talleres creativos, con la finalidad de despertar en los estudiantes el gusto por la expresión oral por medio del juego dramático; Implementación de actividades con el manejo de recursos audiovisuales, demostración teatral por parte de las docentes, selección de guion teatral, elaboración de vestuarios y escenarios; puesta en escena de la obra teatral ante los compañeros.
Fase tres (3): Recopilación de información y sistematización de los resultados; evaluación de los cambios generados a partir de la implementación de la estrategia del juego dramático.

\section{Análisis de resultados}

A continuación, se hará una descripción de cada una de las fases en las que se dividió la metodología teniendo en cuenta los alcances y limitaciones en cada uno de los procesos desarrollados en la misma. La primera fase corresponde a la identificación del problema, dimensión del impacto que genera en los procesos educativos, elaboración de diagnóstico y diseño de los talles del plan operativo.

Fase 1:

Tabla 1 Identificación del Problema, Elaboración de Diagnóstico y Talleres.

\begin{tabular}{|c|c|c|c|}
\hline Actividad & Objetivo & Observaciones relevantes & Pre-Conclusiones \\
\hline $\begin{array}{l}\text { Identificar el } \\
\text { problema }\end{array}$ & $\begin{array}{l}\text { Reconocer las } \\
\text { dificultades de } \\
\text { expresión oral } \\
\text { presentadas } \\
\text { por los } \\
\text { estudiantes } \\
\text { para iniciar en } \\
\text { la búsqueda } \\
\text { de posibles } \\
\text { alternativas de } \\
\text { solución. }\end{array}$ & $\begin{array}{l}\text { Los estudiantes presentan } \\
\text { dificultades con su } \\
\text { expresión corporal, } \\
\text { principalmente en diálogos } \\
\text { formales en donde } \\
\text { interviene un adulto. El } \\
\text { tono de voz utilizado en sus } \\
\text { presentaciones en público, } \\
\text { no es el más adecuado por } \\
\text { lo general es muy suave. } \\
\text { En la pronunciación de } \\
\text { algunas palabras, presenta } \\
\text { dificultad. } \\
\text { No hacen uso de la } \\
\text { expresión corporal. } \\
\text { Demuestran timidez, } \\
\text { inseguridad y miedo } \\
\text { en las conversaciones, } \\
\text { principalmente formales. } \\
\text { Se les dificulta sustentar } \\
\text { sus respuestas debido a un } \\
\text { vocabulario escaso. }\end{array}$ & $\begin{array}{l}\text { Los niños presentan } \\
\text { dificultades en su expresión } \\
\text { oral, lo que dificulta un } \\
\text { proceso de comunicación } \\
\text { exitoso, que a su vez } \\
\text { repercute en su autoestima } \\
\text { y seguridad. } \\
\text { Esta dificultad es más } \\
\text { notoria en conversaciones } \\
\text { formales en las que } \\
\text { interviene un adulto; } \\
\text { cuando el proceso } \\
\text { comunicativo busca que } \\
\text { el estudiante dé cuenta } \\
\text { de sus conocimientos, } \\
\text { experiencias o ideas. }\end{array}$ \\
\hline
\end{tabular}


Se les dificulta sustentar sus respuestas debido a un vocabulario escaso.
La comunicación oral es vital en la transmisión reciproca de conocimientos y construcción colectiva de los mismos, una adecuada comunicación garantiza un mejor aprendizaje y una mayor apropiación de los conceptos.

La comunicación oral es una de las primeras y más usadas formas de transmitir información, quien tiene una adecuada fluidez verbal tendrá mayores oportunidades de liderazgo, y maneja una autoestima y seguridad adecuadas que le permiten desenvolverse de forma exitosa en la sociedad

La palabra es una herramienta valiosísima en los procesos de comunicación, con la palabra se construye y se destruye.

Es la base de los acuerdos

Cuando los estudiantes no logran trasmitir con claridad sus ideas o no encuentran las palabras Identificar Dimensionar el impacto
el impacto que que causa causa en la la expresión comunicación oral en un asertiva de los adecuado estudiantes. proceso de correctas para expresar sentimientos, opiniones o conocimientos, los receptores del mensaje no logran entender el mensaje. El manejo de un tono de voz suave y monótona, comunicación. tiende a aburrir a los oyentes y perder el interés en la comunicación. o el inicio de los conflictos, el valor y utilidad de la palabra toma dimensiones inimaginables.

Por tanto, quien usa un adecuado repertorio - oral, tiene más posibilidades de triunfo en el ámbito académico, personal, profesional y social.

Para que estos procesos comunicativos exitosos se den, se deben iniciar a muy temprana edad cimentando las primeras bases en el hogar, buscando que el niño exprese, se comunique, cree seguridad en sus comunicaciones, no le tenga miedo al fracaso, si no que por el contrario encuentre en las dificultades un motivo más para seguir mejorando y perfeccionando el arte de comunicar. 


\begin{tabular}{|c|c|c|}
\hline $\begin{array}{l}\text { Concurso } \\
\text { de coplas } \\
\text { costumbristas }\end{array}$ & $\begin{array}{l}\text { Identificar las } \\
\text { habilidades y } \\
\text { dificultades } \\
\text { que presentan } \\
\text { los estudiantes } \\
\text { en cuanto a } \\
\text { expresión oral. }\end{array}$ & $\begin{array}{l}\text { Los estudiantes presentan } \\
\text { un tono de voz adecuado, } \\
\text { aunque algunos de ellos } \\
\text { sienten un poco de } \\
\text { inseguridad al recitar en sus } \\
\text { presentaciones en público, } \\
\text { y la expresión corporal es } \\
\text { escasa. }\end{array}$ \\
\hline
\end{tabular}

Esta forma poética, permite

a los estudiantes hacer

un rescate cultural de

términos, escritos y coplas

que han sido incorporadas

en un historial memorístico

importante dentro de cada

familia de esta región, dando un valor popular en la actualidad, en pro del mejoramiento de la expresión oral y corporal.

\begin{tabular}{l}
\hline Elaborar un \\
diagnóstico \\
para \\
identificar \\
conocimientos \\
previos.
\end{tabular}

Identificar

en los estudiantes los conocimientos

que poseen acerca de la expresión oral.

\section{Los estudiantes reconocen} que la comunicación está presente casi en todas las actividades de la vida cotidiana.

Demuestran gusto por ser escuchados.

Reconoce que las emociones se pueden demostrar de forma no verbal.
Los estudiantes son conscientes que un adecuado proceso de comunicación favorece la adquisición y transmisión de conocimientos, las relaciones interpersonales, el desenvolvimiento en cualquier actividad académica, cultural o de la vida cotidiana.

\begin{tabular}{ll}
\hline $\begin{array}{l}\text { Diseño de } \\
\text { talleres } \\
\text { creativos }\end{array}$ & Diseñar \\
& actividades \\
& creativas que \\
& mejoren la \\
& expresión \\
& oral, teniendo \\
& en cuenta los \\
& gustos de los \\
& estudiantes.
\end{tabular}

Luego del análisis de las respuestas dadas por los estudiantes al cuestionario planteado para identificar las actividades que favorecen la expresión oral, preferidas por ellos y de identificar las dificultades más notorias que ellos presentan mediante el concurso de coplas, se decidió tener en cuenta las siguientes: poesía, canciones, trabalenguas, diálogos, mímicas, juego de roles e imitaciones, talleres de expresión corporal.
Luego de identificar las dificultades más notorias en los estudiantes en cuanto a expresión oral, se diseñaron las actividades a trabajar con el fin de mejorar, ojalá en gran medida, la expresión oral de estos estudiantes, cada actividad planteada tiene el objetivo de trabajar una parte específica de la comunicación oral. La poesía trabaja la expresión oral y corporal; trabalenguas con el fin de mejorar la pronunciación y agilidad; canto con el fin de mejorar el dominio de público, su expresión oral y corporal; retahílas con el objeto de lograr en el estudiante mejorar la pronunciación y agilidad verbal; mímicas para mejorar la expresión corporal y perder la timidez; taller de expresión corporal, para mejorar la expresión corporal 
En la segunda fase, se aplicaron los talleres diseñados y se tomaron evidencias fílmicas y fotográficas de cada una de las actividades, para el posterior análisis de los resultados arrojados con la aplicación del plan operativo.

Fase 2:

Tabla 2. Aplicación de talleres

\begin{tabular}{|c|c|c|c|}
\hline Actividad & Objetivo & Observaciones relevantes & Pre-Conclusiones \\
\hline Poesía & $\begin{array}{l}\text { Mejorar sus } \\
\text { habilidades } \\
\text { comunicativas y de } \\
\text { expresión corporal. }\end{array}$ & $\begin{array}{l}\text { Mediante la declamación } \\
\text { de poemas y poesíaslos } \\
\text { estudiantes lograron } \\
\text { avanzar paulatinamente } \\
\text { en la expresión corporal y } \\
\text { la modulación en el tono } \\
\text { de voz. }\end{array}$ & $\begin{array}{l}\text { Esta clase de } \\
\text { representaciones son } \\
\text { manifestaciones de } \\
\text { sentimientos que } \\
\text { realizan las personas, } \\
\text { en este caso los } \\
\text { estudiantes expresan } \\
\text { sus sentimientos } \\
\text { y emociones, al } \\
\text { declamar poesías, } \\
\text { dando prioridad a la } \\
\text { habilidad de expresión } \\
\text { oral. }\end{array}$ \\
\hline Canciones & $\begin{array}{l}\text { Desarrollar en el } \\
\text { estudiante dominio } \\
\text { del público, } \\
\text { expresión corporal } \\
\text { y oral. }\end{array}$ & $\begin{array}{l}\text { Participación de los } \\
\text { estudiantes en un acto } \\
\text { cultural organizado } \\
\text { por la institución, con } \\
\text { la interpretación de } \\
\text { canciones expresaron } \\
\text { mensajes haciendo } \\
\text { uso adecuado de } \\
\text { la expresión oral y } \\
\text { corporal demostrando } \\
\text { progresos en su proceso } \\
\text { comunicativo. }\end{array}$ & $\begin{array}{l}\text { En el momento } \\
\text { que los estudiantes } \\
\text { deciden expresar una } \\
\text { composición musical, } \\
\text { afrontan con más } \\
\text { seguridad la idea de } \\
\text { dirigirse a un público } \\
\text { y dar a conocer ideas } \\
\text { y sentimientos que no } \\
\text { habían manifestado. }\end{array}$ \\
\hline Trabalenguas & $\begin{array}{l}\text { Perfeccionar la } \\
\text { pronunciación y } \\
\text { agilidad verbal. }\end{array}$ & $\begin{array}{l}\text { Los estudiantes } \\
\text { manifiestan una actitud } \\
\text { positiva en el desarrollo } \\
\text { de la actividad, interés y } \\
\text { motivación, sin embargo, } \\
\text { algunos de ellos deben } \\
\text { practicar más ejercicios } \\
\text { de dicción para mejorar la } \\
\text { pronunciación de algunas } \\
\text { palabras. }\end{array}$ & $\begin{array}{l}\text { La idea de recitar } \\
\text { trabalenguas, conlleva } \\
\text { al estudiante a manejar } \\
\text { una pronunciación } \\
\text { compleja y un poco } \\
\text { enredada, lo que hace } \\
\text { que mantenga su } \\
\text { concentración y tono } \\
\text { de voz fuerte, para que } \\
\text { al pronunciar sea fácil } \\
\text { comprender el mensaje } \\
\text { que trae consigo, } \\
\text { también es importante } \\
\text { mencionar que el } \\
\text { estudiante desarrolla su } \\
\text { expresión oral. }\end{array}$ \\
\hline
\end{tabular}




\begin{tabular}{|c|c|c|c|}
\hline $\begin{array}{l}\text { Centro } \\
\text { literario, } \\
\text { retahílas }\end{array}$ & $\begin{array}{l}\text { Mejorar la dicción } \\
\text { y agilidad oral en } \\
\text { los estudiantes. }\end{array}$ & $\begin{array}{l}\text { Es evidente el avance } \\
\text { parcial de los estudiantes } \\
\text { en cuanto a la } \\
\text { pronunciación y agilidad } \\
\text { verbal }\end{array}$ & $\begin{array}{l}\text { En la realización } \\
\text { de estos juegos de } \\
\text { palabras manejados } \\
\text { por rimas y fluidez } \\
\text { verbal, el estudiante } \\
\text { desarrolla un grado una } \\
\text { actitud de expresividad } \\
\text { y seguridad frente a lo } \\
\text { que está recitando. }\end{array}$ \\
\hline Diálogo & $\begin{array}{l}\text { Representar una } \\
\text { conversación entre } \\
\text { sus compañeros, } \\
\text { defiende su punto } \\
\text { de vista y opina } \\
\text { frente al tema } \\
\text { expuesto. }\end{array}$ & $\begin{array}{l}\text { Entablar una } \\
\text { conversación entre } \\
\text { dos o más compañeros } \\
\text { donde propongan un } \\
\text { tema central y las ideas } \\
\text { no se desfasen de este, } \\
\text { manejando una expresión } \\
\text { oral adecuada }\end{array}$ & $\begin{array}{l}\text { La intencionalidad } \\
\text { que se presenta en un } \\
\text { diálogo, es afrontar } \\
\text { un tema junto con } \\
\text { cada punto de vista } \\
\text { que presentan los } \\
\text { participantes y } \\
\text { la manera como } \\
\text { defienden con } \\
\text { argumentos lo que } \\
\text { mencionan, dejando } \\
\text { notar claramente } \\
\text { la expresión oral y } \\
\text { seguridad, para lograr } \\
\text { un acuerdo. }\end{array}$ \\
\hline Mímicas & $\begin{array}{l}\text { Lograr que } \\
\text { el estudiante } \\
\text { reproduzca las } \\
\text { acciones de las } \\
\text { otras personas, } \\
\text { o gestos de un } \\
\text { animal. mejora } \\
\text { su expresión } \\
\text { corporal y gestual y } \\
\text { disminuye el temor } \\
\text { a expresarse ante } \\
\text { un público }\end{array}$ & $\begin{array}{l}\text { Se realizó un ejercicio } \\
\text { de expresión corporal, } \\
\text { siendo evidente que } \\
\text { la mímica como parte } \\
\text { lúdica de la actividad se } \\
\text { transmite un mensaje no } \\
\text { verbal utilizando gestos } \\
\text { e imitación desafiando su } \\
\text { timidez. }\end{array}$ & $\begin{array}{l}\text { Muchas veces } \\
\text { encontramos niños } \\
\text { en las aulas de clase } \\
\text { que, de forma natural, } \\
\text { sienten gusto por imitar } \\
\text { y efectuar acciones que } \\
\text { les permita asemejarse } \\
\text { a un personaje favorito } \\
\text { o un animal. De esta } \\
\text { manera. Es posible } \\
\text { lograr que desarrollen } \\
\text { la habilidad de } \\
\text { expresión oral. }\end{array}$ \\
\hline Juego de roles & $\begin{array}{l}\text { Representar } \\
\text { diferentes } \\
\text { papeles dentro } \\
\text { de situaciones } \\
\text { cotidianas donde } \\
\text { intervienen variadas } \\
\text { posturas, actitudes } \\
\text { y características de } \\
\text { la expresión oral y } \\
\text { corporal }\end{array}$ & $\begin{array}{l}\text { Cada alumno representa } \\
\text { un papel en el que } \\
\text { es necesario tomar } \\
\text { diferentes posturas } \\
\text { de simulación como } \\
\text { intercambiando roles, } \\
\text { permitiendo desarrollar } \\
\text { la creatividad y la } \\
\text { imaginación del } \\
\text { estudiante. }\end{array}$ & $\begin{array}{l}\text { Si viene cierto, el } \\
\text { juego de roles se } \\
\text { distingue como una } \\
\text { estrategia de grupo, es } \\
\text { interesante dejar que el } \\
\text { estudiante ejecute esta } \\
\text { clase de actividades } \\
\text { donde, a manera } \\
\text { de juego, expresa } \\
\text { corporal y oralmente } \\
\text { sus opiniones y } \\
\text { emociones. }\end{array}$ \\
\hline
\end{tabular}




\begin{tabular}{|c|c|c|}
\hline Imitaciones & $\begin{array}{l}\text { Realizar una acción } \\
\text { que otro compañero } \\
\text { esté realizando, } \\
\text { remedando } \\
\text { sus acciones, } \\
\text { precisamente } \\
\text { maneja la expresión } \\
\text { corporal. }\end{array}$ & $\begin{array}{l}\text { En la actividad de } \\
\text { imitación, el estudiante } \\
\text { realiza alguna cosa o } \\
\text { acción copiando la misma } \\
\text { acción que está viendo } \\
\text { representada en alguien, } \\
\text { de tal forma que permite } \\
\text { desarrollar la expresión } \\
\text { corporal en el estudiante. }\end{array}$ \\
\hline
\end{tabular}

\begin{tabular}{ll}
\hline $\begin{array}{l}\text { Taller de } \\
\text { expresión }\end{array}$ & Comunicar frente a \\
corporal & un grupo sus ideas \\
& sin necesidad de \\
& utilizar las palabras, \\
& dando prioridad a la \\
& expresión corporal.
\end{tabular}
El estudiante buscó la manera de transmitir un mensaje fortaleciendo la habilidad de expresión kinésica y proxémica.

Los estudiantes cuando realizan ejercicios de imitación observan todas las características de lo que quieren imitar realizando un estudio previo, por lo tanto, asemeja cada uno de sus comportamientos y refleja una transformación del hecho que va a representar.

Las representaciones como la expresión corporal permiten que el estudiante comunique diferentes tipos de ideas frente a los demás, sin necesidad de utilizar palabras, solo gestos y movimientos, dando manejo apropiado a la expresión corporal.

\begin{tabular}{ll}
\hline $\begin{array}{l}\text { Demostración } \\
\text { teatral por }\end{array}$ & Incentivar a los \\
parte de las & actudiantes por la \\
docentes. &
\end{tabular}

Los niños se mantienen Los niños muestran un alto grado de motivación por este tipo motivados durante toda la actividad.

Demuestran gusto por la actividad.

Entienden, de forma clara, el mensaje que se les deseaba transmitir por medio de la dramatización hecha por medio de títeres.

Piden que se realicen más actividades como esta. de actividades, se logró captar la atención de todo el grupo durante toda la actividad, luego de la actividad se hicieron preguntas para que ellos dieran cuenta de la comprensión del mensaje que se quería dar por medio de la obra teatral, y las respuestas de los estudiantes fueron acertadas demostrando una alto grado de comprensión de la temática tratada. Algunos niños sentían curiosidad porque sabían que sus profesoras estaban detrás del teatrino, pero no las podían ver, eso hizo que en algunos momentos los estudiantes se pararan de sus puestos para intentar ver a sus docentes. 


\begin{tabular}{|c|c|c|c|}
\hline $\begin{array}{l}\text { Puesta en } \\
\text { escena de una } \\
\text { obra teatral }\end{array}$ & $\begin{array}{l}\text { Lograr que los } \\
\text { niños venzan sus } \\
\text { miedos y sean } \\
\text { capaces de actuar } \\
\text { ante un gran } \\
\text { número de público. }\end{array}$ & $\begin{array}{l}\text { La presentación se realiza } \\
\text { teniendo en cuenta las } \\
\text { tradiciones culturales, con } \\
\text { el pedimento de Jorge } \\
\text { Velosa. }\end{array}$ & $\begin{array}{l}\text { Los niños han } \\
\text { logrado superar } \\
\text { en parte la timidez } \\
\text { que se causa las } \\
\text { presentan en público, } \\
\text { han mejorado en su } \\
\text { expresión corporal, } \\
\text { utilizan movimientos } \\
\text { y gestos que ayudan } \\
\text { a entender de forma } \\
\text { clara la intención } \\
\text { comunicativa del } \\
\text { mensaje transmitido } \\
\text { mediante la } \\
\text { dramatización, } \\
\text { demuestran seguridad } \\
\text { en sus actuaciones. } \\
\text { Utilizan un vestuario } \\
\text { adecuado con el tema } \\
\text { representado. }\end{array}$ \\
\hline $\begin{array}{l}\text { Diseño y } \\
\text { presentación } \\
\text { de la obra } \\
\text { teatral ante } \\
\text { toda la } \\
\text { comunidad } \\
\text { educativa }\end{array}$ & $\begin{array}{l}\text { Exponer frente a } \\
\text { los estudiantes, } \\
\text { la metodología } \\
\text { requerida en } \\
\text { la preparación } \\
\text { y ejecución de } \\
\text { una obra teatral } \\
\text { por medio de un } \\
\text { video, para que } \\
\text { analicen cada uno } \\
\text { de los elementos } \\
\text { destacados; } \\
\text { también, puedan } \\
\text { observar cada uno } \\
\text { de los momentos } \\
\text { destacados en la } \\
\text { representación. }\end{array}$ & $\begin{array}{l}\text { Los estudiantes observan } \\
\text { atentamente cada uno } \\
\text { de los aspectos que se } \\
\text { representan en una obra } \\
\text { teatral, argumentan al } \\
\text { respecto y mencionan } \\
\text { algunas características } \\
\text { relevantes frente a } \\
\text { este procedimiento; } \\
\text { descartando cada una de } \\
\text { las dudas e inquietudes } \\
\text { que se puedan presentar. }\end{array}$ & $\begin{array}{l}\text { Cuando se socializa } \\
\text { la representación } \\
\text { de una obra teatral } \\
\text { frente a un grupo de } \\
\text { estudiantes que no } \\
\text { están acostumbrados } \\
\text { a realizar esta clase } \\
\text { de ejercicios, se } \\
\text { consigue que ellos } \\
\text { identifiquen aspectos } \\
\text { que son relevantes y } \\
\text { les pueden ayudar a } \\
\text { manejar el desarrollo } \\
\text { de la expresión } \\
\text { oral, por lo tanto, } \\
\text { van a tener bases } \\
\text { importantes para } \\
\text { preparar su obra } \\
\text { teatral. }\end{array}$ \\
\hline
\end{tabular}

En la tercera y última fase, se recopilan las evidencias o instrumentos de recolección de información. Para esta investigación, en su gran mayoría, se utilizaron filmaciones, ya que estas permitían observar con más detalle los movimientos corporales de los estudiantes, escuchar el tono de voz utilizado por ellos en las diferentes actividades, y aportaron detalles que con otro instrumento de recolección de información no hubiese sido posible observar; posteriormente, se realizó la sistematización de los resultados, los cuales arrojaron avances muy importantes de los niños en su expresión oral. 
FASE 3.

Tabla 3. Recopilación y Análisis de los Resultados

\begin{tabular}{|c|c|c|c|}
\hline Actividad & Objetivo & $\begin{array}{l}\text { Observaciones } \\
\text { relevantes }\end{array}$ & conclusiones \\
\hline $\begin{array}{l}\text { Recopilación de } \\
\text { información y } \\
\text { sistematización de } \\
\text { los resultados }\end{array}$ & $\begin{array}{l}\text { Recopilar y } \\
\text { seleccionar datos } \\
\text { obtenidos a partir de } \\
\text { la implementación } \\
\text { de actividades } \\
\text { diseñadas y } \\
\text { ejecutadas para } \\
\text { analizar las } \\
\text { habilidades orales } \\
\text { y corporales que } \\
\text { se pretende con los } \\
\text { estudiantes. }\end{array}$ & $\begin{array}{l}\text { En la recolección de } \\
\text { videos y fotografías, } \\
\text { se observa que los } \\
\text { estudiantes manejan un } \\
\text { grado de expresión oral } \\
\text { escaso en las primeras } \\
\text { actividades, sienten } \\
\text { inseguridad de expresar } \\
\text { sus ideas y el tono de } \\
\text { voz debe ir mejorando } \\
\text { en cada una de las } \\
\text { demás actividades. } \\
\text { La timidez frente } \\
\text { a un público, se } \\
\text { maneja acorde } \\
\text { con la preparación } \\
\text { de elementos } \\
\text { importantes en el } \\
\text { diseño de actividades, } \\
\text { orientando el proceso } \\
\text { para ir mejorando } \\
\text { paulatinamente en la } \\
\text { ejecución de estas. }\end{array}$ & $\begin{array}{l}\text { Es importante } \\
\text { observar cada } \\
\text { detalle presentado } \\
\text { en la ejecución de } \\
\text { las actividades, } \\
\text { de esta forma la } \\
\text { sistematización se } \\
\text { puede estructurar } \\
\text { en un esquema que } \\
\text { refleje avances, } \\
\text { dificultades } \\
\text { presentadas y } \\
\text { mejoras para } \\
\text { ponerlas en } \\
\text { práctica en } \\
\text { los procesos } \\
\text { educativos con los } \\
\text { estudiantes. }\end{array}$ \\
\hline $\begin{array}{l}\text { Evaluar los } \\
\text { cambios generados } \\
\text { a partir de la } \\
\text { implementación } \\
\text { de la estrategia del } \\
\text { juego dramático }\end{array}$ & $\begin{array}{l}\text { Conjeturar los } \\
\text { avances presentados } \\
\text { en el material } \\
\text { recolectado como } \\
\text { evidencia de la } \\
\text { realización de } \\
\text { las actividades } \\
\text { trabajadas en cada } \\
\text { fase, observar } \\
\text { avances, logros y } \\
\text { dificultades, de esta } \\
\text { forma argumentar } \\
\text { sobre cada aspecto } \\
\text { observado. }\end{array}$ & $\begin{array}{l}\text { Selecciona la } \\
\text { información } \\
\text { recolectada y } \\
\text { aprecia los aspectos } \\
\text { primordiales en } \\
\text { cada actividad } \\
\text { diseñada y trabajada, } \\
\text { de esta manera, } \\
\text { puede establecer un } \\
\text { argumento reflexivo } \\
\text { acerca del proceso } \\
\text { llevado a cabo con los } \\
\text { estudiantes, de acuerdo } \\
\text { con el logro propuesto } \\
\text { en el momento del } \\
\text { diseño del trabajo de } \\
\text { investigación. }\end{array}$ & $\begin{array}{l}\text { Un paso importante } \\
\text { en la ejecución } \\
\text { de un proyecto } \\
\text { es el análisis y } \\
\text { evaluación de } \\
\text { los resultados } \\
\text { presentados, pues } \\
\text { de ahí depende } \\
\text { realizar una } \\
\text { reflexión clara y } \\
\text { precisa de lo que se } \\
\text { trabajó, además se } \\
\text { argumenta el valor } \\
\text { de este, verificando } \\
\text { si obtuvo logros } \\
\text { en los objetivos } \\
\text { propuestos o que } \\
\text { permitió que no } \\
\text { fuera posible } \\
\text { alcanzar lo } \\
\text { estipulado en la } \\
\text { etapa del diseño }\end{array}$ \\
\hline
\end{tabular}


Al comparar los resultados arrojados en la fase 1 del proceso metodológico, en especial la aplicación de la actividad de coplas costumbristas, donde se pudo evidenciar varias de las dificultades de expresión oral y corporal, presentadas por los estudiantes, y los resultados arrojados en la aplicación de la segunda fase del proceso metodológico, se observó el progreso que esta investigación generó en los estudiantes. Ellos mejoraron su tono y modulación de la voz, sobre todo en las presentaciones en público, perfeccionando la trasmisión de sus mensajes y opiniones; utilizando gestos y movimientos corporales que les permitieron darse a entender con mayor claridad y asertividad; hicieron uso del espacio que tienen a su disposición para mantener y cautivar la atención de su público. Como se observó en el análisis de las evidencias audiovisuales tomadas a los estudiantes con el previo consentimiento de sus padres y que reposan en el archivo de evidencias, elaborado por las autoras de esta investigación.

Aun cuando se han logrado avances, queda por mejorar habilidades, como la pronunciación adecuada de algunas palabras. Se ha venido trabajando con trabalenguas y retahílas, pero, aun así, se presentan dificultades en la dicción; de igual manera, sucede con algunos estudiantes que aún no logran utilizar expresiones corporales y gestos en sus procesos comunicativos, especialmente cuando están ante personas de mayor edad que ellos.

\section{Conclusiones}

Con la aplicación de la metodología planteada para esta investigación, se ha evidenciado un progreso significativo en la expresión oral de los estudiantes de grado segundo, ya que la mayor parte del grupo se expresa con naturalidad; los repertorios orales se han enriquecido manifestándose en ellos más seguridad y asertividad en la comunicación.

La aplicación de actividades como coplas, poemas y canciones, han permitido mejorar la expresión corporal $y$, a su vez, realizar un rescate cultural de tradiciones; ya que la mayor parte de este legado cultural logra conservarse en el tiempo a través de la oralidad.

Aunque los resultados obtenidos son satisfactorios, también se han encontrado dificultades en algunos estudiantes, sobre todo con la pronunciación incorrecta de palabras, por falta de vocalización o por conducta imitativa de sus padres o familiares con quienes comparten su cotidianidad.

La práctica de actividades orales como coplas, poemas, retahílas, canciones, trabalenguas, socio dramas, han mostrado que son una herramienta significativa para fortalecer y mejorar la expresión oral, debido que a través de la práctica misma de oralidad es que el estudiante descubre y se concientiza de sus debilidades a la hora de expresarse oralmente, y desde ahí el niño inicia su proceso de mejoramiento.
Con la aplicación de la metodología planteada para esta investigación, se ha evidenciado un progreso significativo en la expresión oral de los estudiantes de grado segundo 


\section{Referencias Bibliográficas}

Cardozo Cuellar, M. Chicue PÁez, C. (2012). Desarrollo de la expresión oral a través de los títeres en el grado primero a y b de la Institución Educativa Agroecológico Amazónico, Trabajo de grado publicado. Universidad de la Amazonia. Florencia (Caquetá). Fernández de la Torriente, G. Libro, como hablar coherente en público.pag.15-35. Biblioteca UPTC.

NúÑEZ Rojas, M., \& NúÑEZ Rojas, J. (2012). El teatro como herramienta didáctica para el desarrollo de la expresión oral en los niños de grado transición de la Institución Educativa Juan Bautista Migani. (Proyecto de grado publicado). Universidad de la Amazonia, Caquetá. Colombia

Navarro Gil, A. (2013). El teatro como estrategia para fortalecer la oralidad, Desarrollado con estudiantes de grado quinto de la institución Educativa Santo Cristo - sede Marco Fidel Suárez. (Trabajo de grado publicado). Universidad de Antioquia, Caucasia, Colombia.

MuÑoz, L, \& PARADA, L. (2015).El teatro pedagógico como herramienta de desarrollo de las habilidades de expresión oral en los niños, a partir de la propuesta de talleres y unidades didácticas aplicadas en el grado sexto del colegio Pierre de Fermat. Corporación Universitaria Minuto de Dios. Bogotá. Colombia. Recuperado de: http://repository.uniminuto.edu:8080/xmlui/bitstream/ handle/10656/3396/THUM_ParadaGarzonLuz_2015.pdf?sequence=1

SÁNCHEZ, L. (2013). El Teatro, actuación creativa, una estrategia para potenciar la competencia comunicativa de los estudiantes de grado tercero de primaria (301) de la Institución Educativa IED Tomás Carrasquilla jornada tarde. Universidad Pedagógica Nacional. Bogotá. Colombia recuperado de: http:// repositorio.pedagogica.edu.co/xmlui/bitstream/handle/123456789/500/ TE-16887.pdf?sequence $=3$

Barón, L \& Muller, O. (2014). La teoría lingüística de Noam Chomsky: del inicio a la actualidad. Fundación Universidad los Libertadores. Bogotá. Colombia. Recuperado de: http://webcache.googleusercontent.com/ search?q=cache:http://www.scielo.org.co/pdf/leng/v42n2/v42n2a08.pdf

Gastón en su libro ¿Cómo hablar correctamente en público recuperado de: http:// www.fudepa.org/fudepaweb/Actividades/FMedia/TecHablar.pdf

Santamaría, A; Gómez, F; Ruiz, F; Boquete M; Vaqueiro, M; Peñasco, M; Moreda L; Comba O; Fernández, M \& Martín, H. (2014). Arriba el telón: enseñar teatro y enseñar desde el teatro. Organizados por el área de Estudos Espanhóis de la universidad de do Minho con la colaboración de la consejería de educación de la embajada de España en Portugal, del instituto de letras de ciencias humanas del centro de investigación. Recuperado de: www.mecd. gov.esportugal-dems-consejerías-exteriores-ortugalpublicaiones-arribaeltelarribaelTelon.pdf. 
Barone, L. (2012). Libro técnicas de la comunicación oral. Pag.49-74. Buenos Arias. Argentina. Categoría primaria. Grupo editorial Clasa. Serie de lineamientos curriculares de artística Ministerio de educación nacional. Bogotá. Magisterio 2000

Konstantin, S. (1957). El trabajo del actor sobre el personaje. Capítulo 5 y 6, Pág., 105-141 Rusia. Grupo Editorial Tomo.

Miretti, M. (2001). El teatro como herramienta didáctica para el desarrollo de la expresión oral en los niños de grado transición de la Institución Educativa Juan Bautista Migani. Pág., 75.

NúÑez, M. \& Núñez, J. Proyecto de grado publicado. Universidad de la Amazonia. Caquetá.

Cervera, J. (sf). Como practicar la dramatización con niños de 4 a 14 años? Editorial del Cardo. Recuperado de: www.biblioteca.org.ar/comentario

Motos \& Tejado, 1996, Citado por Ostos, I (2009). La dramatización en el contexto escolar. ISSN 1988-6047. Recuperado de: http://recursos.salonesvirtuales.com/ assets/bloques/IGNACIO_OSTOS_TORRES02.pdf.

Piaget, J. (1998). Introducción a Piaget: Pensamiento, Aprendizaje y Enseñanza. México: Longman, S.A.

Puyuelo, M. (2012). El teatro como herramienta didáctica para el desarrollo de la expresión oral en los niños de grado transición de la Institución Educativa Juan Bautista Migani. Pág45.Nuñez, M \& Núñez, J. Proyecto de grado publicado. Universidad de la Amazonia. Caquetá.

Zubiria Samper, M (1995), Teoría de las seis lecturas. Mecanismos del aprendizaje semántico. Tomo I: preescolar y primaria. Santa Fé de Bogotá: Fundación Alberto Merani. Editorial Gerardo Andrade M. 